\title{
Resiliencia académica: Comprendiendo las relaciones familiares que la promueven
}

\author{
Academic Resilience: Understanding Family Relationships That Promote it \\ Resiliência acadêmica: Compreender as relações familiares que a promovem
}

Sonia Ilse Salvo-Garrido

Universidad de La Frontera

Departamento de Matemática y Estadística

Temuco, Chile

sonia.salvo@ufrontera.cl

https://orcid.org/0000-0002-9567-2285

José Luis Gálvez-Nieto

Universidad de La Frontera

Departamento de Trabajo Social

Temuco, Chile

jose.galvez@ufrontera.cl

https://orcid.org/0000-0001-7642-8204

Susana San Martín-Parihuén

Universidad de La Frontera

Temuco, Chile

susanasanmartin91@gmail.com

https://orcid.org/0000-0002-9704-811X

Recibido • Received • Recebido: 17 / 08 / 2019

Corregido • Revised • Revisado: 27 / 03 / 2021

Aceptado • Accepted • Aprovado: 23 / 04 / 2021

\begin{abstract}
Resumen. El estudio de la resiliencia académica resulta relevante, por el rol positivo que ejerce sobre el desarrollo educativo y socioemocional de niños, niñas y adolescentes. El objetivo del artículo fue comprender las relaciones familiares que favorecen la resiliencia académica en estudiantes de enseñanza básica, primero a cuarto año, provenientes de contextos vulnerables. La metodología utilizada para dar respuesta a los objetivos, consideró un diseño de teoría fundamentada, ya que se buscó describir y comprender los significados construidos intersubjetivamente por las personas participantes. La selección de quienes participaron se realizó mediante un muestreo teoréticamente guiado, en donde recolección y análisis de datos se realizaron de manera paralela, y la guía principal fue el modelo teórico emergente. La estrategia de recogida de datos consideró la aplicación de 27 entrevistas semiestructuradas a familias vulnerables; para ello se elaboró una guía temática, que contenía los principales tópicos a abordar con las personas participantes, sin un esquema estándar de categorías de respuesta. Los protocolos y procedimientos de análisis de datos se basaron en el paradigma de codificación e incluyó los niveles de codificación abierta, axial y selectiva. Los resultados sugieren que las relaciones familiares basadas en la comunicación, confianza, normas académicas
\end{abstract}


http://doi.org/10.15359/ree.25-2.20

http://www.una.ac.cr/educare

educare@una.ac.cr

estrictas combinadas con apoyo emocional parental favorecen la resiliencia académica. De la misma forma, altas expectativas académicas y compromiso escolar, caracterizado por un componente de afectividad de los padres y madres hacia el colegio, contribuyen a promover la resiliencia académica. Finalmente, se discuten los resultados a partir de la evidencia teórica nacional e internacional disponible, destacando aquellas relaciones familiares saludables que estimulan la resiliencia académica.

Palabras claves: Desarrollo positivo; relaciones familiares; logro académico; resiliencia.

Abstract: The study of academic resilience is relevant to the educational environment because of its positive role in children and adolescents' educational and socioemotional development. This article aimed to understand the family relationships that favor academic resilience in elementary school students, from first to fourth grade, from vulnerable contexts. The methodology used to respond to the objectives considered a grounded theory design since it sought to describe and understand the meanings constructed intersubjectively by the participants. The participants were selected through a guided theoretical sampling, in which data collection and analysis were carried out in parallel, with the main theoretical guide being the emerging theoretical model. The data collection strategy considered the application of 27 semi-structured interviews to vulnerable families, for whom a thematic guide was prepared containing the main topics to be addressed with the participants and without a standard scheme of response categories. Data analysis protocols and procedures were based on coding paradigms and included open, axial, and selective coding levels. The results suggest that family relationships based on communication, trust, and strict academic standards, combined with parental emotional support, favor academic resilience. Likewise, high academic expectations and school commitment, characterized by a component of parents' affection towards school, contribute to promoting academic resilience. Finally, results are discussed from national and international theoretical evidence available, highlighting those healthy family relationships that promote academic resilience.

Keywords: Academic achievement; family structure; positive development; resilience.

Resumo: $O$ estudo da resiliência acadêmica é relevante pelo papel positivo que desempenha no desenvolvimento educacional e socioemocional nas crianças e nos adolescentes. $\mathrm{O}$ objetivo do artigo foi compreender as relações familiares que favorecem a resiliência acadêmica em estudantes do ensino fundamental, do primeiro ao quarto ano, de contextos vulneráveis. A metodologia utilizada para responder aos objetivos foi baseada em um desenho teórico, uma vez que buscou descrever e compreender os significados construídos intersubjetivamente pelas pessoas participantes. A seleção dessas pessoas foi realizada através de uma amostra guiada teoricamente, onde a coleta e análise dos dados foram realizadas em paralelo, sendo principal guia o modelo teórico emergente. A estratégia de coleta de dados considerou a aplicação de 27 entrevistas semiestruturadas às famílias vulneráveis, para as quais foi elaborado um guia temático, que continha os principais temas a serem discutidos com as pessoas participantes, sem um esquema padrão de categorias de resposta. Os protocolos e procedimentos de análise de dados foram baseados no paradigma de codificação e incluíram níveis de codificação abertos, axiais e seletivos. Os resultados sugerem que as relações familiares baseadas na comunicação, confiança, padrões académicos rigorosos combinados com o apoio emocional dos pais fomentam a resiliência acadêmica. Da mesma forma, as elevadas expectativas acadêmicas e o compromisso da escola, caracterizado pelo componente de afetividade das famílias pela escola, contribuem para promover a resiliência académica. Finalmente, os resultados são discutidos com base nas evidências teóricas nacional e internacional disponíveis, destacando-se as relações familiares saudáveis que estimulam a resiliência académica.

Palavras-chave: Desenvolvimento positivo; relações familiares; desempenho académico; resiliência. 


\section{Introducción}

Durante las últimas dos décadas, los sistemas educativos latinoamericanos han vivenciado una serie de reformas a nivel político y estructural (Gómez y Rivas, 2017). No obstante, los resultados académicos medidos a través de pruebas estandarizadas nacionales e internacionales dan cuenta de la falta de eficacia de dichas políticas públicas (Castro Aristizábal et al., 2017). Estos resultados académicos reflejan la falta de equidad en los sistemas educativos latinoamericanos, que se caracterizan por una alta estratificación y fuerte poder explicativo de las condiciones socioeconómicas de las familias (Treviño et al., 2016). Ambos elementos afectan negativamente el rendimiento académico del estudiantado proveniente de los niveles socioeconómicos más bajos, y perpetúan los ciclos de pobreza.

Sin embargo, a pesar de las condiciones adversas que puede enfrentar el estudiantado, en términos de pobreza y vulnerabilidad social, existen niños y niñas que alcanzan rendimientos académicos sobresalientes como sus pares provenientes de familias con situaciones económicas favorecidas, estos alumnos y alumnas han sido denominados como estudiantes resilientes.

En un sentido formal, el Programa para la Evaluación Internacional de Alumnos PISA (Organisation for Economic Co-operation and Development [OECD], 2016), considera estudiantes resilientes cuando obtienen un buen rendimiento académico y provienen de un nivel socioeconómico bajo. Específicamente, los resultados de Chile muestran que un 14,6\% de estudiantes son resilientes entre sus pares que se han categorizado como pobres, mientras que en países como Perú alcanza un 3,2\% y en República Dominicana un 0,4\%. Por otro lado, en países asiáticos como Singapur el 49\% del estudiantado pobre es resiliente.

Esta investigación centra su interés en el concepto de resiliencia académica, un constructo específico y de gran interés para el ámbito educativo (OECD, 2016). La resiliencia académica se refiere a un constructo orientado a los activos, basado en las fortalezas y aspiraciones que se visualizan a través de las acciones del estudiantado frente a la adversidad académica y los beneficios alcanzados (Martin y Marsh, 2009).

\section{Resiliencia académica: Relevancia y definición conceptual}

Un interesante corpus de investigación ha evidenciado que la resiliencia académica favorece el rendimiento académico, la calidad de vida, la salud mental y el bienestar socioemocional (Rodríguez-Fernández et al., 2018).

La resiliencia, en términos generales, presenta diversas definiciones que dificultan consensuar de manera más clara su alcance y dimensiones (Fletcher y Sarkar, 2013), sin embargo, hay relativo acuerdo en considerarla como un proceso dinámico y de naturaleza evolutiva (Masten, 2014), que se construye a partir de las interacciones entre la persona y su contexto. La resiliencia se refiere a los comportamientos que despliega el ser humano frente a condiciones adversas que derivan en una adaptación exitosa. 
http://doi.org/10.15359/ree.25-2.20

http://www.una.ac.cr/educare

educare@una.ac.cr

La resiliencia académica se define como la capacidad que muestran algún estudiantado para superar las adversidades que se presentan de manera aguda o crónica, que amenazan las posibilidades de un adecuado desarrollo educativo (Martin, 2013). De este modo, se puede visualizar cómo estudiantes que muestran un rendimiento disminuido, logran mejorar y prosperar en el contexto escolar (Martin y Marsh, 2006).

Martin y Marsh (2009) proponen un instrumento de medición de la resiliencia académica, en una escala que operacionaliza la resiliencia académica a partir de cinco factores: autoeficacia, control, planificación, baja ansiedad y persistencia. Existen características y actitudes específicas entre el estudiantado resiliente, entre las que se encuentran la perseverancia, fuerza de voluntad y entusiasmo en las actividades que realizan (Chrysani et al., 2017).

Rouse (2001) señala que el estudiantado resiliente cree más en su propia habilidad y en su capacidad de tener el control de su vida escolar, necesita menor apoyo externo en comparación con sus pares no resilientes. También se siente menos agotado o falto de energía, ya que la resiliencia académica actúa como factor protector frente al cansancio emocional (Fínez Silva y Morán Astorga, 2014). Por otra parte, Morales (2008) realizó un estudio donde encontró que la adaptabilidad, determinación y habilidades metacognitivas son un rasgo fundamental para alcanzar sus metas escolares.

La resiliencia académica depende de los diferentes niveles involucrados en el desarrollo de una persona; la familia, escuela y grupo de pares, el contexto comunitario, el contexto sociocultural y valórico, y el cronosistema (Bronfenbrenner, 1986). Gran cantidad de estudiantes cuenta con diversos factores de riesgo que generalmente conducen al fracaso o al mal desempeño en tareas cognitivas o académicas; no obstante, algunos alumnos y alumnas superan los obstáculos pudiendo demostrar resiliencia académica, por lo que no es suficiente solo centrarse en el desarrollo de las habilidades del pensamiento (Kuldas et al., 2015). Por consiguiente, el presente trabajo centra su atención en el rol de la familia que considera a los padres, madres, parientes así como a las personas adultas que cumplen funciones parentales y el desarrollo de la resiliencia académica por parte del estudiantado, sin desconocer que existen otros factores que están involucrados en el proceso de desarrollo de estas características que manifiesta una parte del estudiantado.

\section{Familia y resiliencia académica}

El apoyo que brinda la familia a sus hijos e hijas permite disminuir la aparición de conductas riesgosas, las relaciones familiares sanas propician que jóvenes en riesgo desarrollen su talento y potencial (Abdul Kadir et al., 2012). Se ha observado que la formación de autonomía, comunicación y control conductual, genera en los hijos y las hijas mayor autoestima, resiliencia y habilidades socioemocionales (Ruvalcaba-Romero et al., 2016). 
Las familias cumplen un rol fundamental en el desarrollo de la resiliencia de los hijos y las hijas, ya que luego de enfrentar situaciones difíciles centran su atención en el presente y el futuro, se niegan a permitir que el trauma afecte las relaciones familiares, logran que las experiencias negativas los alentaran hacia el progreso y alcanzan que sus hijos e hijas obtengan un mayor desempeño en el contexto escolar (Grotberg, 2001).

Específicamente, la resiliencia académica se relaciona con una buena relación afectiva y de cuidado de los padres y madres hacia los hijos e hijas (Chrysani et al., 2017). La familia figura como un factor protector de niños y niñas que residen en entornos en que existe pobreza y violencia, ya que la orientación y apoyo que le pueden brindar los padres y madres fomentan la resiliencia y rendimiento académico en estudiantes en riesgo (Rojas, 2015).

Las creencias y valoración positiva que atribuyen los padres y madres al proceso de escolarización de sus hijos e hijas, se asocian con el compromiso escolar y las altas expectativas de los hijos y las hijas, aspectos que generan una mejora en el rendimiento académico (Chrysani et al., 2017). También la percepción que la familia tiene de sus hijos e hijas influye en cómo enfrentan los desafíos en la escuela, fortalecen la resiliencia y el autoconcepto académico (Rautiainen et al., 2015).

En la misma línea de investigación, Räty et al. (2014) estudian la resiliencia académica del estudiantado desde la mirada de los padres y madres y los resultados de los hijos e hijas evaluando sus habilidades y competencia en la escuela. Las personas autoras señalan que la percepción de los padres y madres tuvo efectos significativos en las evaluaciones de las competencias verbales y cognitivas de la niñez, tendiendo a mantenerse estables en el tiempo, independiente de los resultados de sus hijos e hijas.

Otro estudio (Li, 2017) plantea que la supervisión de los padres o madres, la participación y el reconocimiento de la escuela se asocian positiva y significativamente con la resiliencia académica $y$, de manera negativa, con el conflicto individual y el bajo compromiso escolar. En cuanto a los estudios de resiliencia académica en estudiantes rurales, (Li y Yeung, 2019) plantean que los resultados concuerdan con las evidencias halladas en occidente, vale decir, refuerzan la importancia de los recursos de competencia familiar y escolar de la niñez, a pesar de las condiciones desfavorables que enfrentan en su cotidianeidad. Se puede considerar que las expectativas de los padres y madres cuando son visualizadas por los hijos e hijas les sirven para comprender que pueden romper el ciclo intergeneracional de la pobreza.

El alumnado que cuenta con mayor riesgo de abandono escolar percibe que sus padres y madres poseen menos competencias en el desarrollo personal y educativo, así como menor resiliencia (Martín Quintana et al., 2015), mientras que los padres y madres de estudiantes resilientes académicamente monitorean a sus hijos e hijas a través de sus vínculos sociales, teniendo una participación más cercana en la educación de sus hijos e hijas (Çelik, 2017), se suma que las características de cohesión familiar y estabilidad ejercen una influencia considerable, ya que la gran mayoría de los adolescentes y las adolescentes que participaron en la investigación crecieron en una familia con padre y madre a la vez (Chrysani et al., 2017). 
http://doi.org/10.15359/ree.25-2.20

http://www.una.ac.cr/educare

educare@una.ac.cr

Otro estudio muestra que las personas inmigrantes resilientes académicamente que contaron con el apoyo y la orientación de los padres, madres y la familia; que recibieron ayuda en las tareas, apoyo financiero y moral, y orientación para obtener mejores calificaciones en la escuela, lograron mayor rendimiento académico (Kumi-Yeboah, 2020). Según Löfgren y Löfgren (2017), los padres, madres crean importantes expectativas en los hijos e hijas que observan su potencial y éxito; para el estudiantado es relevante complacerse a sí mismo y sentirse satisfecho, mientras paralelamente las madres y los padres están contentos con sus esfuerzos y resultados. El éxito en la escuela es visualizado como un vehículo para tener una buena vida en el futuro, por lo que los padres y madres los incentivan a través de la narración de sus experiencias como estudiantes.

Ahora bien, considerando la evidencia precedente, se confirma la significativa asociación entre la resiliencia académica y el cuidado, ya que, de lo contrario, el estudiantado expresa baja autoestima y depresión (Neal, 2017). Por otro lado, cuando se observan los facilitadores del éxito educativo y la asistencia a la universidad entre jóvenes en adopción temporal (en hogares de guarda), los resultados de la investigación sugieren que la estabilidad y las relaciones positivas con personas mentoras adultas facilitan las transiciones y refuerzan las conexiones emocionales que afectan el éxito educativo y la asistencia posterior a la secundaria (Strolin-Goltzman et al., 2016).

De este modo, investigar sobre resiliencia académica y las relaciones familiares cobra relevancia, sobre todo en estudiantado desfavorecido y que recibe menos apoyo familiar, ya que, gran cantidad, está atrapado en ambientes vulnerables que limitan enormemente las posibilidades de superar desventajas tan complejas como la pobreza. Este estudio se enfoca particularmente en estudiantes vulnerables y tiene como objetivo comprender las relaciones familiares que promueven la resiliencia académica desde la perspectiva de padres, madres, personas apoderadas y así identificar aquellos aspectos que favorecen la resiliencia educativa y cuáles son las dimensiones que merecen mayor atención por parte de las comunidades escolares para poder tomar decisiones locales informadas sobre este factor protector. En este sentido, la resiliencia académica actúa como un activo para el desarrollo, si se considera que Chile tiene un sistema educativo altamente estratificado, donde la situación socioeconómica de la familia tiene un alto impacto en el rendimiento del alumnado (OCDE, 2016; Treviño et al., 2016), este estudio permitirá comprender cómo este factor protector es vivenciado por las propias familias y, así, pretende contribuir a romper con los ciclos de desventaja en el proceso educativo a través de favorecer que el estudiantado cuente con habilidades y el potencial para desafiar el sistema actual.

\section{Método}

\section{Diseño}

La investigación utilizó un diseño de teoría fundamentada (Strauss y Corbin, 2002). Este diseño de investigación sienta sus bases en el interaccionismo simbólico, dado que busca la comprensión de los significados construidos intersubjetivamente, que desarrollan interpretaciones del mundo de los sujetos en función de sus discursos. 


\section{Trabajo de campo}

El trabajo de campo se realizó utilizando una metodología mixta secuencial cuantitativa y cualitativa. En primer lugar, se seleccionaron estudiantes resilientes mediante técnicas estadísticas multivariantes aplicadas a las bases de datos de la prueba SIMCE -Sistema de Medición de Calidad de la Educación- de Chile. Posteriormente se seleccionaron aquellos establecimientos con mayor proporción de estudiantes resilientes provenientes de tres macrozonas de Chile (norte, central y sur).

Luego se tomó contacto con los establecimientos educativos y se solicitó autorización para contactar a las familias del estudiantado resiliente. La aplicación fue realizada por dos profesionales pertenecientes al ámbito de la educación y tres que conformaron el equipo de investigación asociado al proyecto. En términos territoriales y considerando la larga extensión de Chile, el trabajo de campo se dividió en tres macrozonas: sur, centro y norte del país. Siguiendo el diseño de teoría fundamentada, se utilizó un muestreo teoréticamente guiado (Strauss y Corbin, 2002), en donde trabajo de campo y el análisis de datos se realizaron en forma paralela -tópico que se abordará nuevamente en el apartado de análisis de datos-, esto permitió mejorar el contenido de la guía temática e incrementar la calidad de los datos recolectados. El proceso de muestreo teorético finalizó una vez que las categorías se saturaron, esto fue al aplicar 27 entrevistas a familias provenientes de 18 establecimientos públicos y particulares subvencionados de todas las macro zonas del país.

\section{Técnicas y estrategias de recogida de datos}

Para alcanzar los objetivos de investigación se aplicaron 27 entrevistas semiestructuradas, basadas en una guía temática que favoreció la indagación sobre los significados de las vivencias de las personas participantes. Se entrevistó a un miembro de la familia, dependiendo de la estructura familiar, fueron seleccionados para participar: padres, madres, parientes, así como a las personas adultas que cumplían funciones parentales.

En primera instancia, se elaboró la guía temática que contenía los principales tópicos a abordar con las personas participantes, la que fue sometida a juicio de cinco personas expertas con conocimientos en el área de estudio. Las preguntas no contaban con un esquema fijo de categorías de respuesta; profundizaban en temáticas que emergieron espontáneamente desde las respuestas de quienes participaron, lo que posibilitó que surgieran contenidos que no habían sido contemplados por el equipo de investigación.

\section{Procedimiento}

Para la aplicación de las entrevistas semiestructuradas, se tomó contacto con los directores y directoras de los establecimientos y se solicitó permiso para acceder a la muestra. Con el objetivo de resguardar los principios éticos, se realizaron reuniones informativas donde 
http://doi.org/10.15359/ree.25-2.20

http://www.una.ac.cr/educare

educare@una.ac.cr

se explicaron los objetivos y alcances del estudio, para finalmente aplicar un consentimiento informado que resguardó los siguientes principios éticos: voluntariedad, resguardo de la información, confidencialidad y anonimato de las personas participantes.

\section{Análisis de datos}

Para el análisis de datos se utilizó el paradigma de codificación propuesto por la teoría fundamentada (Strauss y Corbin, 2002) y el software Atlas ti 7.5. Como método para la interpretación de los textos, se utilizó inductivamente el muestreo comparativo constante, que consistió en la identificación y comparación de códigos a partir de las subunidades discursivas más relevantes de los documentos primarios o entrevistas. En un nivel de abstracción mayor, se utilizó como técnica la redacción de memos, que permitieron la incorporación de notas y reflexiones teóricas emergentes durante el proceso de codificación.

Siguiendo los protocolos y procedimientos de la teoría fundamentada (Strauss y Corbin, 2002), el análisis de datos se desarrolló a partir de cinco etapas. La primera incluyó la transcripción de las entrevistas. La segunda etapa de codificación abierta, que abarcó la dimensión descriptiva del análisis, consideró un microanálisis de las entrevistas, revisando línea a línea su contenido, en este sentido los datos fueron segmentados, examinados y comparados, luego desde un trabajo inductivo se identificaron los principales conceptos y significados que los fragmentos contenían, esta etapa concluyó con la selección de los códigos más relevantes y fusión de códigos con temáticas similares. La tercera etapa del análisis denominada codificación axial, identificó las familias de códigos, también denominadas categorías discursivas y las relaciones explicativas entre los códigos de cada categoría -insumo para la construcción de los mapas de redes-. Una cuarta etapa fue la codificación selectiva, en donde los investigadores enjuiciaron la centralidad y relevancia de las categorías para responder la pregunta de investigación. Finalmente, en la quinta etapa del proceso, se dispuso la información en redes o network que graficaron las relaciones entre códigos y categorías, que expresaban condiciones, contextos y dimensiones de la resiliencia académica.

\section{Resultados}

Para el equipo de investigación resultaba relevante contar con la perspectiva de las familias y poder estructurar así la red de relaciones que favorecen la resiliencia académica, ya que ambas se vinculan estrechamente. Es importante aclarar que los resultados que se presentarán a continuación, en ningún caso refieren procesos lineales ni causales, así también señalar que entendemos a las familias como sistemas sociales complejos, que pueden manifestar dinámicas contradictorias, por ejemplo, familias con hijos o hijas que presentaban rasgos resilientes e hijos o hijas que, habitando bajo el mismo techo, sometidos o sometidas a la misma estructura de normas y valores académicos, no presentaban dichos rasgos. En este sentido, las relaciones 
entre categorías y subcategorías que se analizarán deben ser entendidas desde lógica anterior, asumiendo que la resiliencia se construye con base en procesos dinámicos entre el individuo y su contexto. Los resultados que se presentan a continuación han identificado las siguientes categorías de análisis: superación de la adversidad, compromiso escolar y relaciones familiares.

\section{Superación de la adversidad}

La categoría superación de la adversidad tiene que ver con la capacidad de sobreponerse y superar situaciones de vida que podrían ser negativas para el desarrollo del estudiantado (Figura 1). De acuerdo con los resultados, las situaciones adversas varían según cada realidad familiar; sin embargo, los discursos de las familias tienden a estabilizarse a partir de las siguientes subcategorías: ausencia de la madres o el padre o ambos, uso excesivo de alcohol de algún miembro de la familia, enfermedad física del estudiante y metas educativas. La superación de la adversidad no se logra rápidamente, es un proceso que se vive y como todo proceso tiene aspectos tanto positivos como negativos; en este sentido, los problemas familiares los hacen sufrir frustración y molestias, esto genera peleas y conflictos entre los miembros del grupo familiar. Sin embargo, la superación de dichos problemas implica algo positivo, sobreponerse y comprender que una condición familiar o personal que dificulta el desarrollo académico puede transformarse en la oportunidad de adquirir herramientas para ser más tolerantes al sufrimiento y enfrentar con actitud positiva los problemas educativos que, en términos comparativos, son menos graves que los acontecidos en sus trayectorias de vida.

Una de las subcategorías de la superación de la adversidad es la ausencia de la madre o el padre o ambos. En el caso de nuestros datos encontramos distintas situaciones que generan que el estudiantado no viva con su padre o su madre, entre ellas destaca, la separación de la pareja, muerte o abandono. En casos extremos, cuando ambos, padre y madre, están ausentes, generalmente su rol es asumido por familiares directos, como abuelos, abuelas o tíos y tías de las personas menores. En otras situaciones, como la separación de la pareja, los roles pueden ser asumidos por nuevos integrantes tales como padrastros o madrastas. La situación de adversidad generada por ausencia del padre o la madre o ambos precipita el desarrollo de las características de resiliencia académica, como la tolerancia ante el sufrimiento, una actitud positiva para resolver problemas académicos y la motivación para alcanzar sus metas educativas.

La subcategoría de metas educativas tiene que ver con el logro de objetivos académicos a mediano y largo plazo, y se basa en alcanzar un buen rendimiento académico para facilitar el proceso de movilidad social y mejorar su condición socioeconómica. Esta subcategoría se relaciona con las expectativas académicas de la familia, que pueden oscilar desde esperar que los hijos e hijas desarrollen carreras militares, hasta metas académicas ligadas a estudiar en las mejores universidades del país. Aquellos hijos o hijas que tienen metas educativas claras y fijadas con antelación al término de los procesos académicos -ingreso a la Universidad-son los que generan resultados académicos sobresalientes, en comparación con los hijos e hijas con indecisón, que cambiaban constantemente sus metas académicas. 
http://doi.org/10.15359/ree.25-2.20

http://www.una.ac.cr/educare

educare@una.ac.cr

Mi hija Francisca, nunca supo que quería estudiar, le fue mal en la enseñanza media, le fue mal en la PSU [prueba de selección universitaria], rendimos juntas la PSU y me fue mejor a mí que a ella... yo le decía "pero Francisca ¿qué quieres estudiar tú?", entonces ahí llegó a ser socióloga... ¿Entiende? En cambio, mis otras dos hijas (estudiantes resilientes), lucharon muchisimo para ser ingenieras y quedar aceptadas en las mejores universidades del país, porque no es fácil. Carla obtuvo buen puntaje en la PSU, la Paula igual, ¿me entiende? Y sin preuniversitario, Claudia obtuvo muy buenas notas igual, entonces ellas tenían clarísimo lo que querían estudiar. (Entrevista N. ${ }^{\circ} 12$ )

Figura 1: Categoría Superación de la adversidad

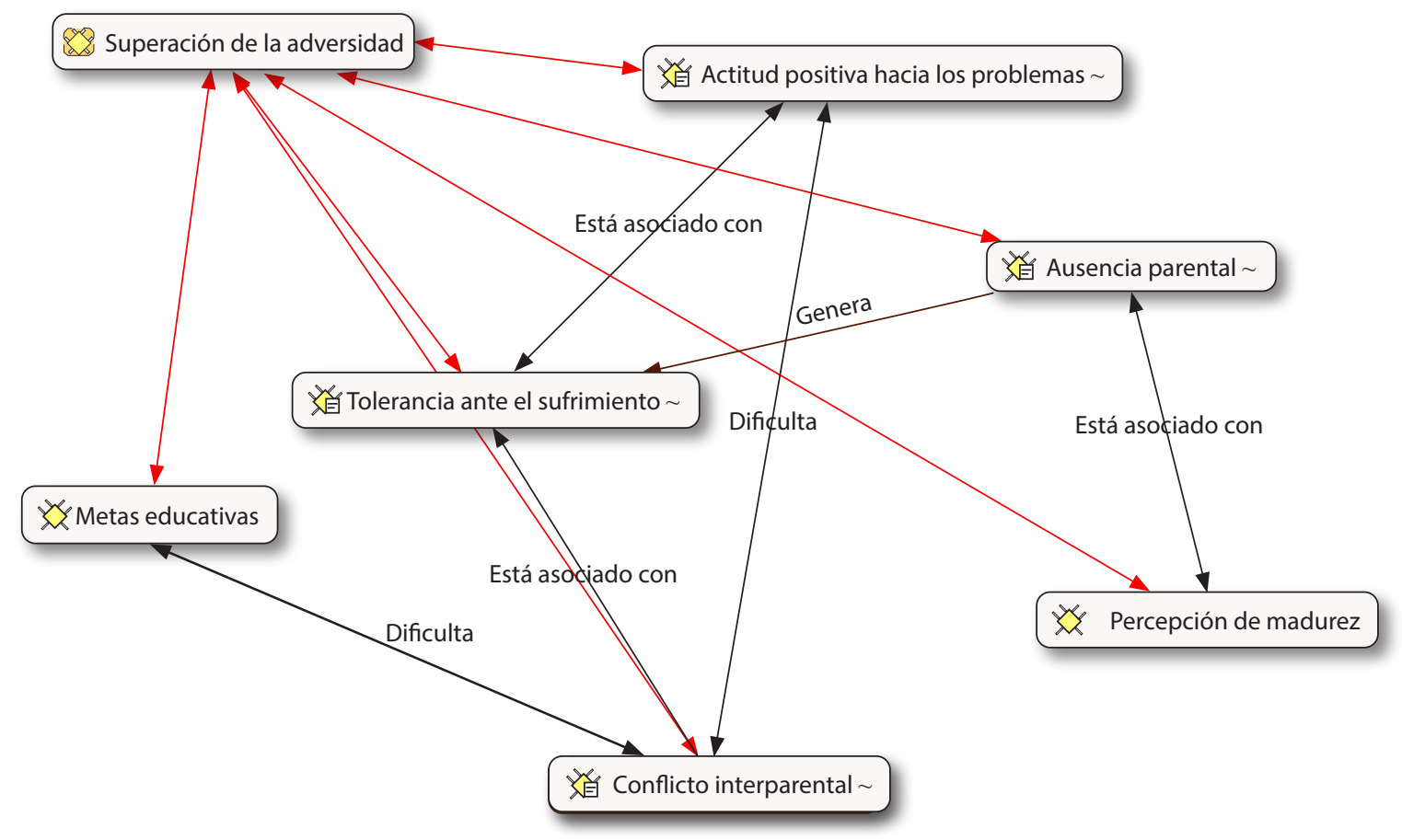

Nota: Elaboración propia.

\section{Compromiso escolar}

En cuanto a la categoría de compromiso escolar, se refiere a los vínculos y lazos que se generan entre la familia y la escuela, en estos casos la familia presenta una actitud positiva hacia la escuela, dado que se siente y está implicada en la educación de sus hijos e hijas (ver Figura 2). La familia participa activamente en las actividades del colegio, en términos prácticos se preocupa por comprar los útiles escolares; colaborar en las tareas escolares y asistir a los horarios de atención de reforzamiento escolar con sus hijos e hijas; la familia se siente conectada afectivamente con la escuela. 
http://doi.org/10.15359/ree.25-2.20

La categoría de compromiso escolar tiene como subcategoría la motivación, que se basa en el sentimiento positivo o negativo de realizar tareas escolares. El compromiso escolar y la motivación varían de acuerdo con el grado de apoyo y supervisión que proporcionan los padres y las madres, y la retroalimentación que entreguen el personal docente en cuanto a las tareas académicas encomendadas. Por ejemplo, un estudiante que recibe apoyo y ayuda para realizar sus tareas -indirectamente está bajo la supervisión de sus padres y sus madres- y además es retroalimentado y supervisado por el personal docente, efectúa sus quehaceres con agrado en un ambiente favorable; en cambio una estudiante que vivencia un escaso apoyo y supervisión parental, combinado con nulas o escasas retroalimentaciones del personal docente, siente que realiza las tareas sin estímulo favorable que compense el trabajo y tiempo invertido.

Lo que mi hijo reclamaba, era que en varias oportunidades que él hacía sus tareas, pero algunos profesores no las revisaban. Entonces él sentía que se esforzaba y perdía el tiempo, en algunas ocasiones me decía, mamá para que voy a hacer mis tareas si no las van a revisar. (Entrevista $\mathrm{N}^{\circ} 18$ )

La familia se siente orgullosa de su hijo o hija y de los logros académicos que estos pueden alcanzar. Es relevante distinguir que la persona familiar responsable o más implicada en el proceso educativo siente orgullo al entender que su esfuerzo favorece un buen desempeño académico en el niño o niña. Este es un proceso complejo, ya que no es posible establecer, como causa, que las características de estudiantes resilientes se deben únicamente al apoyo de la familia, sino a un proceso circular de retroalimentación.

Figura 2: Categoría Compromiso escolar

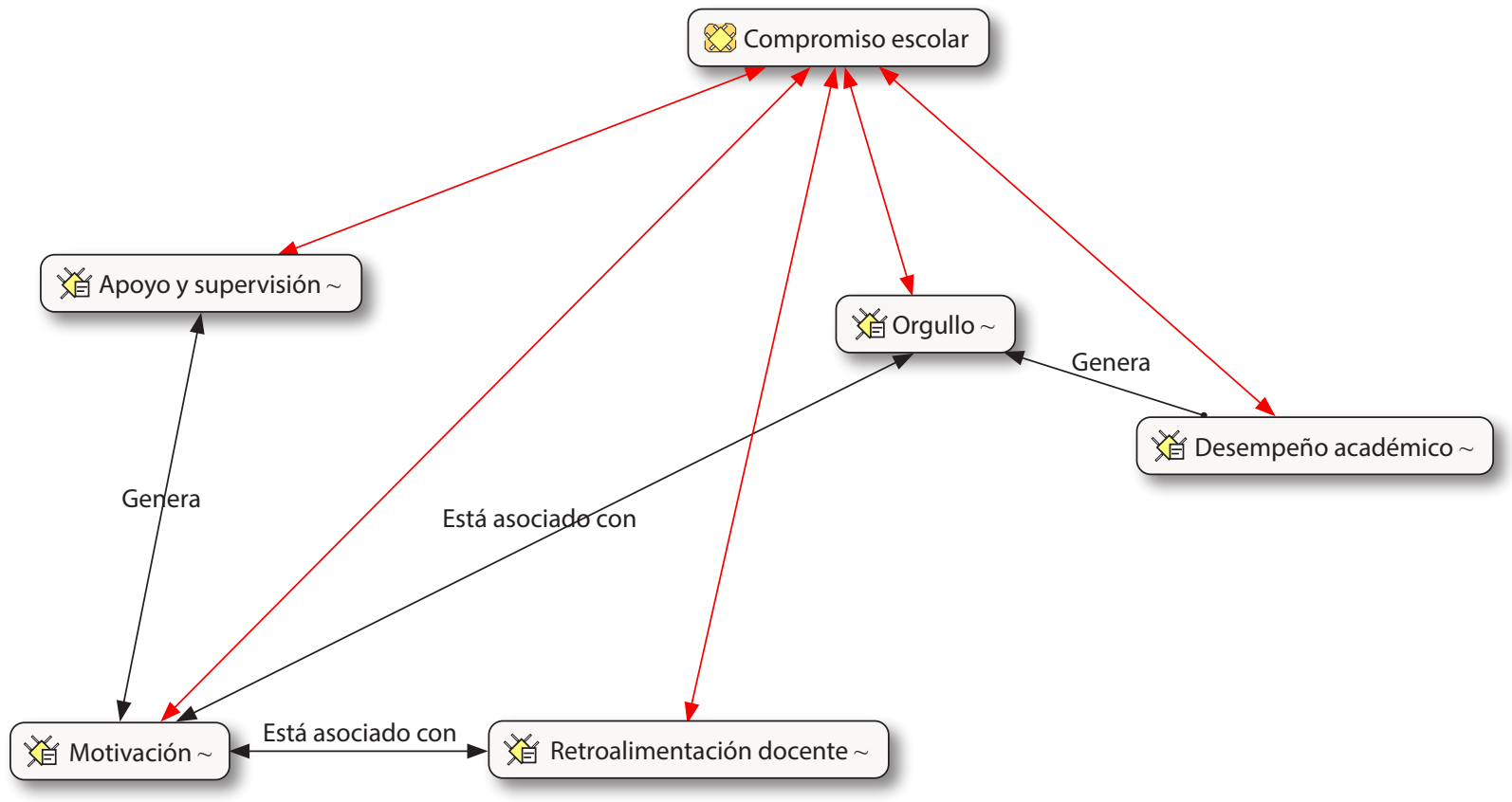

Nota: Elaboración propia. 
http://doi.org/10.15359/ree.25-2.20

http://www.una.ac.cr/educare

educare@una.ac.cr

\section{Relaciones familiares}

Las relaciones familiares que favorecen la resiliencia académica tienen, como base de interacción, la comunicación fluida de miembros de la familia, caracterizada por una escucha activa hacia los hijos y las hijas, y la inclusión de su opinión en la toma de decisiones (Figura 3). Estos aspectos son combinados, a su vez, con procesos de control y supervisión parental, que confieren a la interacción un tono estricto, pero democrático.

Nosotros tenemos mucho diálogo familiar, no sé permiten los teléfonos en la mesa, tengo mucho control con el internet, no soy permisiva, para nada, mi hija sus permisos, colegio casa, casa-colegio y alguna parte todo nosotros lo hacemos en familia, los cinco, tratamos de no tener ningún tema tabú en nuestra familia. (Entrevista $\mathrm{N}^{\circ} 25$ )

Directamente asociada a la comunicación familiar emerge la subcategoría de revelación, que consiste en la capacidad desarrollada por los hijos e hijas para proporcionar información sobre las actividades realizadas en el tiempo libre con amistades y sobre los problemas que enfrentan cotidianamente. Dicha dimensión se basa en la confianza generada, al interior de la familia, para abordar tanto problemas académicos como de la vida diaria.

Me van contando lo que han vivido durante el día, durante la tarde o cosas que han pasado en el colegio, cosas bonitas, feas, grandes, pequeñas, me las cuentan igual, ellas solas. (Entrevista $N^{\circ} 6$ )

Otra de las subcategorías que emerge a partir de los discursos de las familias es la aplicación de normas y límites, pero llevadas a una esfera académica, considerando el resguardo de espacios para el estudio, horario para realizar tareas y herramientas tecnológicas. El estudiantado resiliente desarrolla labores académicas con energía, es capaz de sobreponerse y hacer sus trabajos a pesar de sentir cansancio. Otro elemento a tener en cuenta es la autonomía con que desarrollan sus actividades, no necesariamente deben tener el control parental.

La dimensión de expectativas familiares se refiere a las posibilidades de movilidad social ascendente. El estudiantado resiliente está expuesto a una mayor proporción discursos de sus posibilidades de acceder a la universidad y a cursar una carrera profesional de pregrado, en ninguno de los casos se señaló que la máxima expectativa pudiese ser el postgrado. Otra de las subcategorías señaladas por las familias tiene que ver con dedicarse a una carrera militar.

Ella tiene muy claro lo que quiere estudiar, siempre lo ha tenido muy claro, son dos alternativas, hacer el servicio militar o ser profesora de lenguaje o de educación física, ella lo tiene claro. $Y$ mis expectativas, yo la apoyo al cien por ciento nada más, porque tengo claro lo que ella quiere, siempre lo ha tenido claro... y yo sé que ella va a llegar allá, porque mi hija es capaz. (Entrevista $\mathrm{N}^{\circ} 13$ ) 
Figura 3: Relaciones familiares

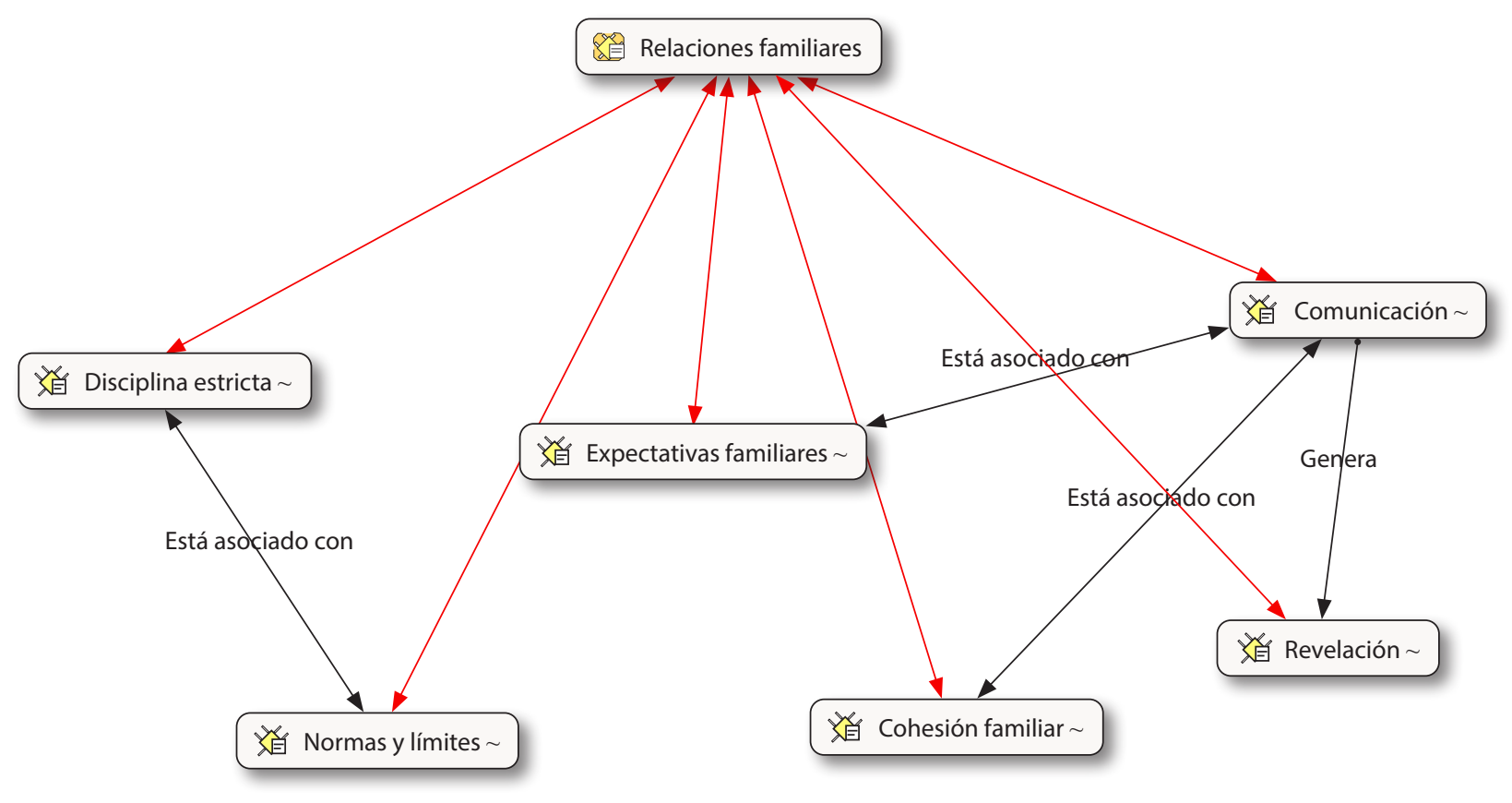

Nota: Elaboración propia.

\section{Discusión}

El propósito de este artículo fue comprender las relaciones familiares que promueven la resiliencia académica desde la perspectiva de padres, madres, personas apoderadas de estudiantes de primero a cuarto años de enseñanza básica de Chile. Los resultados permiten concluir que las relaciones familiares basadas en una adecuada comunicación y un estilo parental basado en el uso de normas académicas combinadas con un alto apoyo emocional parental contribuyen a promover la resiliencia académica.

Los hallazgos de este estudio sugieren que el alumnado resiliente ha desarrollado la capacidad de sobreponerse y superar las situaciones adversas, aspectos que estarían asociados con dificultades de estructura familiar, concretamente con la ausencia de uno o ambos progenitores. En este sentido, estos resultados difieren de los obtenidos por quienes plantean que las características familiares de cohesión, estabilidad y estructura biparental ejercen gran influencia (Chrysani et al., 2017), pues estos hallazgos sugieren que la resiliencia académica no depende solamente de la estructura familiar. 
http://doi.org/10.15359/ree.25-2.20

http://www.una.ac.cr/educare

educare@una.ac.cr

Como equipo de investigación hemos observado que la resiliencia académica depende, en mayor medida, de un soporte emocional adecuado, que en los casos de abandono del padre o la madre o ambos puede ser proporcionado por la familia extendida, abuelos, abuelas, tíos, tías, padrastros o madrastras, o por personas adultas que cumplen funciones parentales. Ello, a su vez, coincide con investigaciones de niños y niñas que han crecido en instituciones y que, a pesar de no estar con su familia, recibieron apoyo emocional de una persona significativa (Strolin-Goltzman, et al., 2016).

En relación con la superación de la adversidad, destaca el esfuerzo que utilizan para enfrentar los desafíos académicos. Al respecto se observa que el estudiantado resiliente cree en su propia habilidad para resolver los problemas académicos, lo cual es concurrente con los planteamientos de Rouse (2001). Los hallazgos de este estudio plantean que el estudiantado resiliente presenta mayor tolerancia al sufrimiento, aspecto que le permite enfrentar con actitud positiva los desafíos educativos, dado que la resiliencia académica actúa como factor protector frente al cansancio emocional (Finez Silva y Morán Astorga, 2014).

En cuanto a las relaciones familiares que promueven la resiliencia académica, destaca como hallazgo la dimensión de estilos de crianza familiar. La comunicación con los referentes parentales es un factor protector, porque estimula mayor autonomía y habilidades socioemocionales en los hijos y las hijas. Estos resultados son consistentes parcialmente con el estudio de Ruvalcaba-Romero et al., (2016), pues en términos procesuales, nuestros hallazgos amplían esta perspectiva, al proponer que una adecuada comunicación familiar estimula que los hijos y las hijas revelen, a sus padres, sus madres o referentes parentales, información sobre actividades cotidianas en el ámbito académico, que pueden oscilar desde problemas sociales, hasta actividades realizadas en el tiempo libre con el grupo de pares en el ámbito escolar. Este hallazgo sugiere que la adecuada comunicación genera confianza y, a su vez, esta confianza eleva la revelación, que se constituiría como un factor protector de la resiliencia académica y adecuados resultados académicos.

También surge, de manera relevante, la capacidad de los padres, las madres y personas significativas de establecer normas o límites, lo cual considera diferentes acciones para colaborar con el estudiantado en la organización de su tiempo y espacio en torno al estudio, lo que no significa necesariamente una supervisión constante en la ejecución de sus tareas, ya que, para ello, el alumnado contaría con la autonomía necesaria que se vincula con su éxito académico (Li y Yeung, 2019). En relación con los resultados de nuestro estudio, las normas y límites resultan particularmente importantes, actúan como factor protector en contextos de pobreza y favorecen la resiliencia académica.

Así también, padres, madres o personas apoderadas tienen fuertes lazos con la escuela, evidencian un significativo compromiso escolar, es decir, se mantienen activos y activas en cuanto a su participación en actividades ligadas a que el estudiantado logre o mantenga sus 
notas y consiga los aprendizajes esperados según su grupo etario. Tal como plantean Chrysani et al. (2017), esto se ligaría a la valoración positiva del proceso de escolarización de las personas adultas. Por su parte, el estudiantado experimenta motivación intrínseca para efectuar sus quehaceres escolares, por lo que busca colaboración en sus padres o sus madres frente a una dificultad que surja en los estudios. De esta manera, los padres y las madres generan expectativas que sirven de motivación para sus hijos e hijas (Löfgren \& Löfgren, 2017), las que, en su mayoría, están ligadas con ser profesionales, ya sea obteniendo un título en la universidad, o perteneciendo a las fuerzas armadas.

En relación con las limitaciones, es posible señalar que la muestra del estudio, si bien considera un amplio espacio territorial, no es representativa de Chile. Sin embargo, el énfasis de este diseño de investigación no estaba puesto en la generalización, sino en la representación teórica y social de las familias con alumnos y alumnas resilientes, por lo cual fue muy importante la densidad de discurso que cada entrevista proporcionó para responder la pregunta de investigación. Este muestreo consideró incorporar procedimientos de análisis de datos desde el inicio de la investigación, procedimiento que obligó al aumento de la muestra hasta lograr que las familias no aportaran nada nuevo respecto del objeto de estudio, este principio es conocido como saturación de información.

En cuanto a futuras líneas de investigación, se debería profundizar la comprensión sobre los mecanismos por los cuales la resiliencia académica promueve un adecuado clima escolar, para responder de mejor manera lo sugerido por la bibliografía respecto de las relaciones sociales al interior de los establecimientos educativos y cómo la resiliencia estimula el éxito académico (Escalante Mateos et al., 2021; Gálvez- Nieto et al., 2020).

\section{Reconocimientos}

Este artículo reporta resultados parciales del proyecto FONDECYT No. 1160429.

\section{Declaración de Material complementario}

Este artículo tiene disponible, como material complementario:

-La versión preprint del artículo en https://doi.org/10.5281/zenodo.4041895

\section{Referencias}

Abdul Kadir, N. B., Rahim, S. A., Mustapha, Z., Abdul Muutalib, M. H., Kee, C. P. y Mohamed, R. H. (2012). External assets as predictors of positive emotions among at-risk youth in Malaysia. Asian Social Work and Policy Review, 6(3), 203-217. https://doi.org/10.1111/j.17531411.2012.00071.x 
http://doi.org/10.15359/ree.25-2.20

http://www.una.ac.cr/educare

educare@una.ac.cr

Bronfenbrenner, U. (1986). Ecology of the family as a context of human development: Research perspectives. Developmental Psychology, 22(6), 723-742. https://doi.org/10.1037/0012$\underline{1649.22 .6 .723}$

Castro Aristizábal, G., Giménez, G. y Pérez Ximénez de Embún, D. (2017). Desigualdades educativas en América Latina, PISA 2012: Causas de las diferencias en desempeño escolar entre colegios públicos y privados. Revista de Educación, 376, 32-59. 10.4438/1988-592XRE-2017-376-343

Çelik, Ç. (2017). Parental networks, ethnicity, and social and cultural capital: The societal dynamics of educational resilience in Turkey. British Journal of Sociology of Education, 38(7), 1007-1021. https://doi.org/10.1080/01425692.2016.1218753

Chrysani, A., Kalogerakis, P. y Katsis, A. (2017). The road to resilience: Breaking the cycle of disadvantage. Educational Journal of the University of Patras UNESCO Chair, 4(1), 71-82. https://doi.org/10.26220/une.2785

Escalante Mateos, N., Fernández-Zabala, A., Goñi Palacios, E. y de-la-Fuente-Díaz-de Cerio, I. I. (2021). School climate and perceived academic performance: Direct or resilience-mediated relationship? Sustainability, 13(1), 1-14. https://doi.org/10.3390/su13010068

Fínez Silva, M. J. y Morán Astorga, C. (2014). Resiliencia y autoconcepto: Su relación con el cansancio emocional en adolescentes. International Journal of Developmental and Educational Psychology, 6(1), 289-295. https://doi.org/10.17060/ijodaep.2014.n1.v6.746

Fletcher, D. y Sarkar, M. (2013). Psychological resilience: A review and critique of definitions, concepts, and theory. European Psychologist, 18(1), 12-23. https://doi.org/10.1027/10169040/a000124

Gálvez-Nieto, J.L., García, J. A., Vera-Bachmann, D., Trizano-Hermosilla, I.y Polanco, K. (2020). Análisis de clases latentes multinivel del clima escolar: Factores individuales, familiares y comunitarios. Revista de Psicodidáctica, 25(2), 85-92. https://doi.org/10.1016/j.psicod.2020.01.001

Gómez, G. y Rivas, M. (2017). Resiliencia académica, nuevas perspectivas de interpretación del aprendizaje en contextos de vulnerabilidad social. Calidad en la educación, 47, 215-233. https://doi.org/10.4067/S0718-45652017000200215

Grotberg, E. H. (2001). Resilience programs for children in disaster. Ambulatory Child Health, 7(2), 75-83. https://doi.org/10.1046/j.1467-0658.2001.00114.x

Kuldas, S., Hashim, S. y Ismail, H. N. (2015). Malaysian adolescent students' needs for enhancing thinking skills, counteracting risk factors and demonstrating academic resilience. International Journal of Adolescence and Youth, 20(1), 32-47. https://doi.org/10.1080/026 $\underline{73843.2014 .973890}$ 
Kumi-Yeboah, A. (2020). Educational resilience and academic achievement of immigrant students from Ghana in an urban school environment. Urban Education, 55(5), 753-782. https://doi.org/10.1177/0042085916660347

Li, H. y Yeung, W.-J. J (2019). Academic resilience in rural chinese children: Individual and contextual influences. Social Indicators Research, 145(1), 703-717. https://doi.org/10.1007/ s11205-017-1757-3

$\mathrm{Li}, \mathrm{H}$. (2017). The 'secrets' of Chinese students' academic success: Academic resilience among students from highly competitive academic environments. Educational Psychology, 37(8), 1001-1014. https://doi.org/10.1080/01443410.2017.1322179

Löfgren, H. y Löfgren, R. (2017). Grades in the eyes of our parents: A narrative approach to educational resilience in pupils' stories of getting their first grades. Nordic Journal of Studies in Educational Policy, 3(2), 165-178. https://doi.org/10.1080/20020317.2017.1343624

Martin, A. J. y Marsh, H. W. (2006). Academic resilience and its psychological and educational correlates: A construct validity approach. Psychology in the Schools, 43(3), 267-281. https:// doi.org/10.1002/pits.20149

Martin, A.J.y Marsh,H.W.(2009). Academic resilience and academic buoyancy: Multidimensional and hierarchical conceptual framing of causes, correlates and cognate constructs. Oxford Review of Education, 35(3), 353-370. https://doi.org/10.1080/03054980902934639

Martin, A. J. (2013). Academic buoyancy and academic resilience: Exploring 'everyday' and 'classic' resilience in the face of academic adversity. School Psychology International, 34(5), 488-500. https://doi.org/10.1177/0143034312472759

Martín Quintana, J. C., Alemán Falcón, J. A., Marchena Gómez, R. y Santana Hernández, R. (2015). El contexto familiar del alumnado en riesgo de abandono escolar temprano según la tipología familiar. Profesorado. Revista de Currículum y Formación de Profesorado, 19(3), 246-263. https://recyt.fecyt.es/index.php/profesorado/article/view/43648

Masten, A. S. (2014). Invited commentary: Resilience and positive youth development frameworks in developmental science. Journal of Youth and Adolescence, 43(6), 1018-1024. https://doi.org/10.1007/s10964-014-0118-7

Morales, E. E. (2008). Academic resilience in retrospect: Following up a decade later. Journal of Hispanic Higher Education, 7(3), 228-248. https://doi.org/10.1177/1538192708317119

Neal, D. (2017). Academic resilience and caring adults: The experiences of former foster youth. Children and Youth Services Review, 79, 242-248. https://doi.org/10.1016/j. childyouth.2017.06.005 
http://doi.org/10.15359/ree.25-2.20

http://www.una.ac.cr/educare

educare@una.ac.cr

Organisation for Economic Co-operation and Development. (2016). PISA 2015 results excellence and equity in education (Vol. 1). https://www.oecd-ilibrary.org/education/pisa-2015results-volume-i 9789264266490 -en

Räty, H., Kasanen, K. y Rautiainen, R. (2014). Educational resilience from parental perspective. Journal of Educational and Developmental Psychology, 4(1), 1-9. https://doi.org/10.5539/ jedp.v4n1p1

Rautiainen, R., Räty, H. y Kasanen, K. (2015). Do parents' and teachers' views of educational resilience matter? Educational Studies, 41(5), 471-475. https://doi.org/10.1080/03055698 .2015 .1043981

Rodríguez-Fernández, A., Ramos-Díaz, E., Ros Martínez de Lahidalga, I. y Zuazagoitia Rey-Baltar, A. (2018). Implicación escolar de estudiantes de secundaria: La influencia de la resiliencia, el autoconcepto y el apoyo social percibido. Educación XX1, 21(1), 87-108. https://doi. org/10.5944/educxx1.20177

Rojas, L. F. (2015). Factors affecting academic resilience in middleschool students: Acasestudy. Gist Education and Learning Research Journal, 11,63-78.https://doi.org/10.26817/16925777.286

Rouse, K. A. G. (2001). Resilient student's goals and motivation. Journal of Adolescence, 24(4), 461-472. https://doi.org/10.1006/jado.2001.0383

Ruvalcaba-Romero, N. A., Gallegos-Guajardo, J., Caballo, V. E. y Villegas-Guinea, D. (2016). Prácticas parentales e indicadores de salud mental en adolescentes. Psicología desde el Caribe, 33(3), 223-236. https://doi.org/10.14482/psdc.33.3.9485

Strauss, A. y Corbin, J. (2002). Bases de la investigación cualitativa. Técnicas y procedimientos para desarrollar la teoría fundamentada. Editorial Universidad de Antioquia.

Strolin-Goltzman, J., Woodhouse, V., Suter, J. y Werrbach, M. (2016). A mixed method study on educational well-being and resilience among youth in foster care. Children and Youth Services Review, 70, 30-36. https://doi.org/10.1016/j.childyouth.2016.08.014

Treviño, E., Fraser, P., Meyer, A., Morawietz, L., Inostroza, P. y Naranjo, E. (2016). Informe de resultados terce: Factores asociados. Unesco. http://archivos.agenciaeducacion.cl/8 Informe internacional_de_resultados_Factores asociados_TERCE 2013.pdf 